\title{
Analysis and Design by Agent based MaSE Methodology: A Case Study
}

\author{
Farhad Soleimanian \\ Gharehchopogh \\ Department of Computer \\ Engineering, Science and \\ Research Branch, Islamic \\ Azad University, West \\ Azerbaijan, Iran
}

\author{
Behnam Zebardast \\ Department of Computer \\ Engineering, Science and \\ Research Branch, Islamic \\ Azad University, West \\ Azerbaijan, Iran
}

\author{
Esmail Amini \\ Department of Computer \\ Engineering, Science and \\ Research Branch, Islamic \\ Azad University, West \\ Azerbaijan, Iran
}

\begin{abstract}
Multiagent Systems Engineering (MaSE) Methodology is one of old object-oriented methodology which supports the development process and is established based on the development of the object-oriented software engineering methods and their adjustment with the agent view. Some characteristics of the agent like autonomy, creativity and preactivated are not paid attention. The agents are supposed as simple software processes which cooperate to obtain a certain goal. There are two basic phases in MaSE: analysis and design. The analysis phase concentrates on specializing the agent's roles, their duties and interactions. In design phase, matters such as diagrams and conversations class are introduced. The all steps in MaSE are implemented by graphic tools, agent Tool. This tool (agent Tool) covers all the steps of MaSE methodology design and analysis. In this article, we have covered the MaSE methodology based on a practical experience. The reason of choosing the chain store system is that it has the necessary characteristics like customer and seller autonomic agent technology and it is easier to identify and understand the analysis and design steps.
\end{abstract}

\section{Keywords}

MaSE Methodology, Design, Software, Chain Store, Agent Tools.

\section{INTRODUCTION}

An agent is an encapsulated computer system that is situated in some environment and that is capable of flexible, autonomous action in that environment in order to meet its design objectives [3]. In recent years, utilizing the agents has been increased significantly in making computer system. The agent technology can make it possible to create systems with characteristics like: high complexity of the software, being open, distributed data and control sources and also high flexibility. How to analyze the issue, the agents view unity in agent-oriented systems, managing these organizational relationships using agent's intelligence and autonomy cause to simplify the establishment of a complicated and open system based on agent. Paying attention to these characteristics of agent based systems; we can account them as next generation of software systems [1]. The main objective of the agentoriented software engineering is to make methodology, tools and possibilities in order to facilitate the development and maintain these agent based software in a cheaper way. In addition, the outcome software is flexible and using it is easier and also has high quality and efficiency.

There was an urgent requirement to a new harmonic engineering with the agent view. It caused to develop the agent-oriented software engineering from object-oriented software engineering because the object-oriented methodology was not able to reply the requirements of the agent based software $[2,3]$. The difference between the agent based methods and object based methods is that an agent has all the traits of an object, but the agent based methods have More standard social relationships which prepare a reciprocal relation ability to relate with other agents. It creates an autonomy which gives the agent a decision making power in system states without any supervisor or external control. When there are some duties should be done by one agent at some time, it helps the system to react immediately and act concurrently toward environmental changes.

So, it can be said that the agent based methods are more active to simulate the objects around us, but the object based methods con not simulate a lot of aspects of the agent based systems since they don't have the necessary simulation facilities. There for, the requirement to new techniques and methods special for agent based systems analysis and design was introduced. The characteristics of the agents cause their flexibility which comes from these agents ability to react to the environmental changes. Following is the objectives and the improvement of these objectives and tasks result in the agent strength. These qualities in complicated systems and environment with errors are very fruitful. In this way, various methodologies were created to design and analyze the agent based systems [4]. These methodologies can be divided in two groups. The first category is the methodologies developed based on the extending methods of object-oriented software engineering methods and their compatibility with the agent view. The examples of these methodologies are Gaia and MaSE [5, 6, 7, and 8].

The second category is methodologies which are the extension of the knowledge engineering methods. MAS-Common KADS is one of these methodologies [9, 10]. Wood and Loach [11] suggest the MaSE. It is one of the important methodologies in agent based software engineering systems. It is assessed and developed practically, it is used as an analysis and design method of systems based on agent and it is the development of the object-oriented methods. 
The life time of this system, from defining step to designing step, is performed based on MaSE by identifying an electronic chain store system. The all steps of design and analysis in agent Tool [12] are performed to support the MaSE methodology, so we will have a practical experience which makes the all steps analysis and design of MaSE easier. Therefore, it can be possible to perform any other system by this agent-oriented software engineering method and have a brighter understanding of the agent resulted in this issue improvement.

In section 2, we have explained the MaSE methodology in order to know the concept and the structure of MaSE methodology, because it is the base of our work. In section 3, seven steps of analyzing and designing of the electronic chain store system performed based on MaSE methodology and diagrams in agent Tool are shown. In section 4, we will express the future works and the conclusion of this paper.

\section{THE AGENT BASED MASE METHODOLOGY}

MaSE methodology which is known as a method of design and analysis of the agent based systems are the development of the object-oriented methods. They were introduced by Wood and Loach [11]. This methodology doesn't see the agents as the autonomous, creative and reactive existences, but it sees them like simple software processes and the agents in this methodology cooperate to obtain the same goal. Indeed the goal of MaSE is to lead the designer from the initial system specification to the implemented agent system [11, 12]. As it was discussed, the task base in MaSE methodology survey is practical experiences resulted from system identification. Here, the electronic chain store systems with the normal complexity and volume have been chosen and they have the essential characteristics to implement with the agent technology.

The normal volume and complexity of the electronic chain store system cause that we know and become familiar with the initial concepts of MaSE methodology and also it facilitates the possibility of design, analysis and implementation of the system completely.

MaSE is known as one of the methodologies of the agentoriented software engineering systems. It implements the software engineering from the definition matter to the implementation stage. It goes through the analysis and design stage in seven steps [12]. This method covers the whole steps of MaSE methodology design and analysis by supporting the graphic tool of agent Tool. It shows all the steps in the form of diagrams. Fig. 1 shows the whole steps of MaSE methodology. We will explain these steps during the chosen system analysis and design.

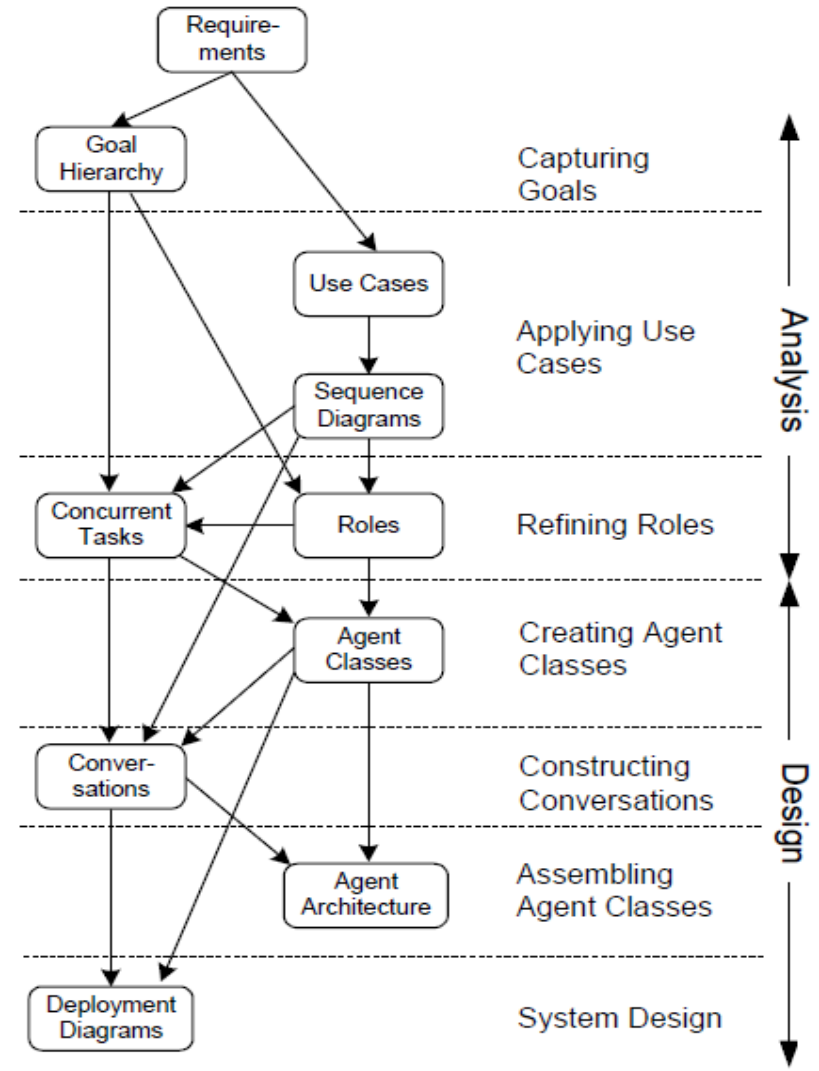

Fig1. MaSE Methodology $[12,13]$

The system we want to implement as an agent-oriented should have the necessary characteristics of the agent-oriented technology. It means the considered software system should have the main traits of one agent in creating multi agent software systems. These characteristics are like autonomy and smart interplay in order to implement all these seven MaSE methodology steps composed of two phases of analysis and design in this system frame. We study the chain store system to show the design and analysis phases of the methodology in this system.

\section{THE ELECTRONIC CHAIN STORE SYSTEM: A CASE STUDY}

In this paper, we want to design a system by MaSE methodology. This system will have all aspects of this methodology and also cover it, so we chose the electronic chain store system for implement so as to show the agents roles in the form of diagrams designed by the agent Tool, but the cases which make chain store system suitable to implement based on the agent technology are:

1. The main characteristics of the electronic system sell and buy participants are their autonomous behavior and reactions. Each buyer or seller can take part in the system autonomously and also participate in gaining their goals, sell and buy work, automatically. The relationship between different parts occurs only through information and messages exchanges.

2. Another characteristic is the seller and buyer agent's activeness. The buyer agent goal is to get standard product with low price and the seller agent wants to sell the product 
with high price and gets more benefits which this shows each agent's activeness and efficiency.

3. It is essential for the system seller to have the ability of making relationship with unknown buyers of the system, because the buyers are not known before entering the system and they enter the system at the time of program implement. Therefore, the requirement is for relationship to unknown individuals in other characteristics of electronic chain store system.

4. In environment of electronic system, the buyer agents, against to the seller agent, reply to the events by messages which this shows each agent's being reactive.

5. In system, there is nothing in the name of individual's ownership of one person. The buyers entered to the system can be the possession of different people, while the owner of the seller agent is apart from the buyer agents in system.

6. The buyer and the seller agents have the social ability it means each agent, seller or buyer, interacts with each other to get their goals. The buyer agent's social ability implicates having relationship and being in harmony with the seller agent.

\subsection{The Chain Store System Analysis}

In this section, we analyze our system made of three steps of Capturing Goals, Applying Use Cases and Refining Roles. These steps have some sections too. We draw the considered parts using agentTool v1.8.2 [14].

\subsubsection{Capturing Goals (Requirements Definition)}

The first step of system analysis in MaSE method is Capturing Goals. In this methodology, the goal is a secession of practical requirements or non-practical ones. In this methodology the Requirements are observed from the system point of view. This step has two parts itself. In first part, analyzer finds the goals in system level from the Requirements characteristics. The next part, he gives them structure and the goal Hierarchy will be made as a tree. Our system which is chain store system contains cases like the buyer request of product, preparing the buyers list for the customers every day, the chief customers conversation with the seller in order to agree on a price apart from the price told by the seller to buy the product, the products price list preparation separately by seller for the chief customers. They have a password and can enter the section and the matters implement are done by smart agents. Since the first prices of the products are considered as the determined prices, if no one requests for a special product, its price will cut down. If its expiration time finishes, the seller has to put it away and if a product becomes extinct by any reason, shortage of production in country or import decline, the seller will sell it by several percent more than before.

The customers who buy a lot of products and distribute it in the city are known as the main (chief) customers. The price of buying products by them is different with other customers and at the time of conversation with the seller. They buy the products paying attention to the inflation and its sale in a day cheaper and is different compared to other customers who buy to obviate their needs. Considering these Requirements, we design and analyze our system. The Goal Hierarchy diagram based on these Requirements has been presented as the Fig. 2.

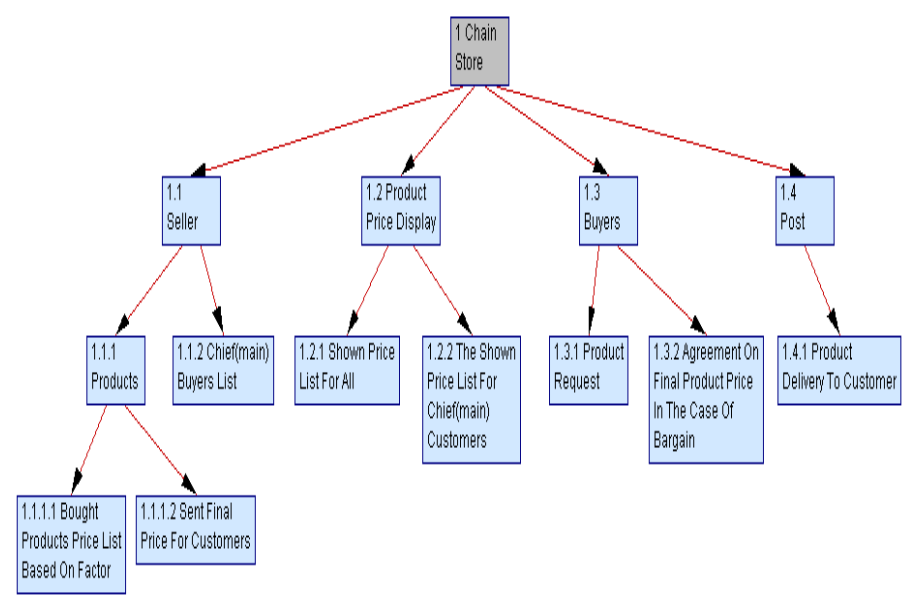

Fig2. Goal Hierarchy Diagram of electronic chain store system

\subsubsection{Applying Use Cases}

The second step of system analysis in MaSE methodology is the Applying Use Cases. The necessary roles and also relationship between these roles are specified in this step. This step has two sections itself, in first section called the use cases; we identify the scenarios and the use cases in order to specify the roles, interactions and system inner relationships. In second section, we draw the Sequence diagram based on the events dynasty occurred in use cases. Indeed the use cases show the logical reciprocal reaction of different roles of the system with each other. To minimize the amount of sent and received messages between the systems, we use the Sequence Diagrams.

Fig. 3 shows a sequence diagram where the buyer is conversing with the seller but because of none existing product the seller ignore the buyer request.

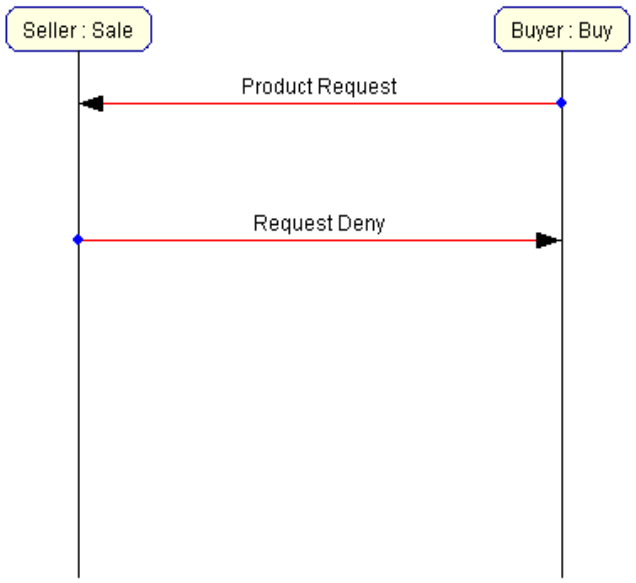

Fig3. Sequence Diagram of the product request deny 
But in Sequence Diagram shown in Fig. 4 which buyer request a product from seller and he does not agree with the specified price. He asks the seller to decline the demanded product price and the seller accepts he offers another price at.al last they get an agreement on the price. The buyer receives the product by the store post. This expresses the autonomy of the buyer and the seller agents and also social interaction.

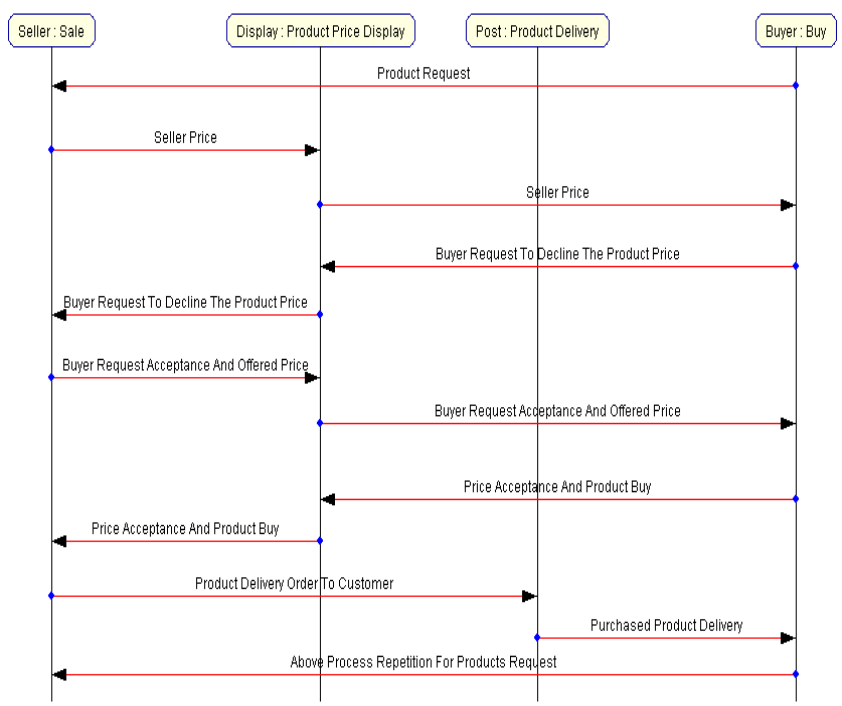

Fig4. Sequence Diagram of the product purchase by the customer

\subsubsection{Refining Roles}

This is the last step in system analysis. It has two parts, in the roles part, there are some roles which respond the certain goals in first step of analysis. Each goal is usually shown by a role, but it is possible to give a set of related goals to one role. In the section of concurrent tasks, roles make a set of tasks together. These tasks can explain how to gain the related goals to each role. Tasks are identified by a position diagram. Therefore, the tasks model done to achieve the goal is drawn. In Fig. 5, each role has several tasks. In this diagram, for instance, the role of seller consists of tasks of the product storage, selling, and the chief buyers list. The selling task is responsible for the updating the list of the chief buyers in order to omit and add the buyers and updating list of the products storage for omitting and adding the products in storage. The seller role has also the selling task, interacting and conversing with the buying task of the buyer role. As we can see, these tasks help to gain the related goal with that role.

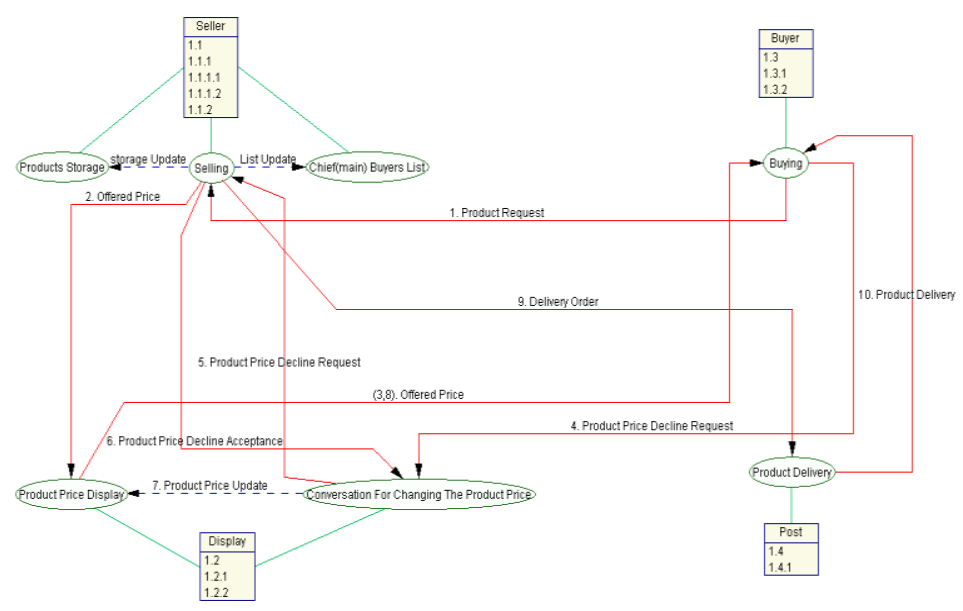

Fig5. Chain store system Role Diagram

Fig. 6 shows the buying task model of the buyer role. It can be seen in Fig. 6, if the price of a product exceeds the permitted limit or if the request of some products be more than number of products existing in storage, the operation will fail otherwise the buyer converses with the seller and waits for the seller respond. The seller checks the rate of benefits based on the buyer offered price and answers him.

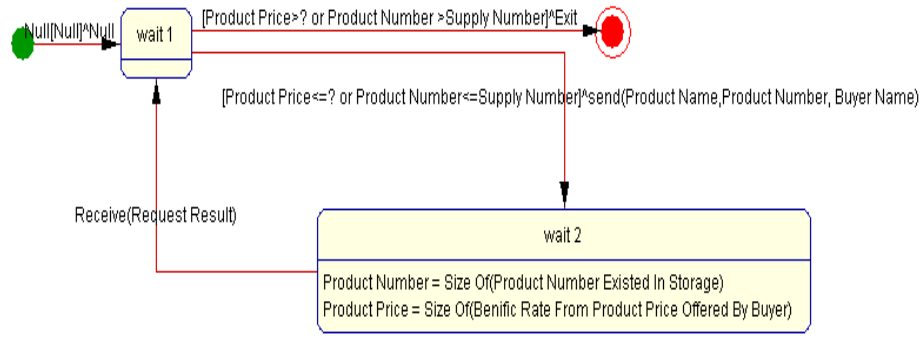

Fig6. Buying Task Panel in chain store system

\subsection{The Chain Store System Design}

In the section, we design our considered system which is composed of four steps: Creating Agent Classes, Constructing Conversations, Assembling Agent Classes and System Design. These steps have their own stages too. We draw the considered parts using agent Tool v1.8.2 [14]. The chain store system design is done by analysis steps.

\subsubsection{Creating Agent Classes}

The first step of designing is Creating Agent Classes. The Agent Class is defined by two characteristics. First in the class, the agents are specified based on the roles determined goals. The next part, discussion will be made between the agents in high levels which are identified based on external communication of the roles. This forms the agent classes' diagram. Fig. 7 shows the agent classes' diagram. In Fig.7, the buyer agent and the seller agent interact to agree on the offered price. 


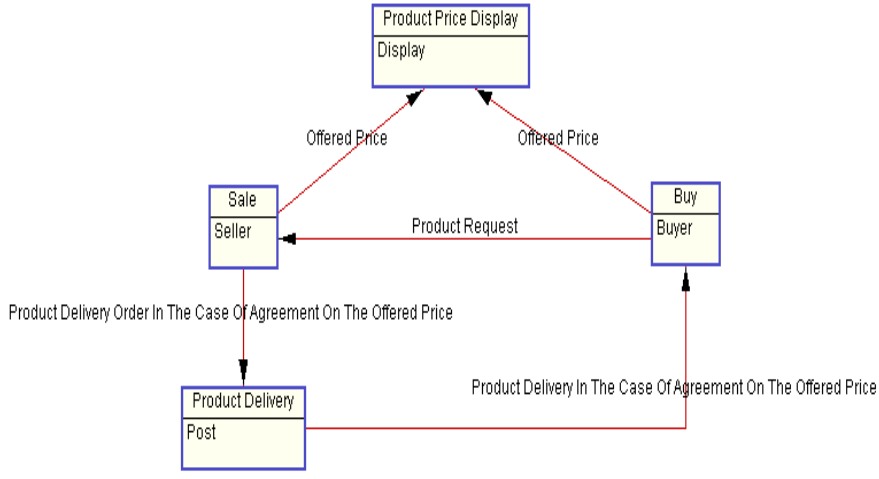

Fig7. Agent Classes Template Diagram in chain store system

\subsubsection{Constructing Conversations}

In constructing conversations step, the goal is to model an agent inner position at the time of receiving the message. To conversation Conversations, we use UML state diagrams and the below cases should be modeled in Conversations.

1- A message is being received causes to pass from one state to another state.

2- The action which will be active as soon as receiving the message.

3- Necessary conditions to pass from one state to another state.

4- A message sent at the time of passing from one state to another state.

Fig. 8 shows the product request constructing conversations by the customer. If the price of a product exceeds a special limit or if the request of some products is more than the number of that products in storage it will cause the buyer to exit the system. If the condition is the opposite of that, the buyer will converse with the seller. If they get an agreement the seller will accept his request. If they do not agree with each other (on the price they both will exit the system.

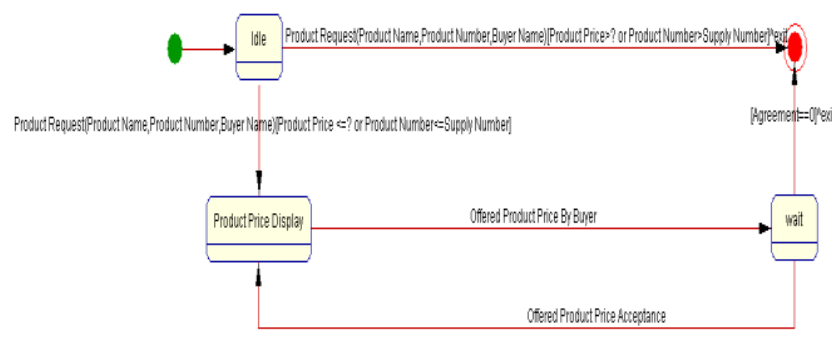

Fig8. Product request Constructing Conversations Diagram by customer

\subsubsection{Assembling Agent Classes}

After designing the whole the class of the agents it is turn to tiny design of the sub structures. For this reason, MaSE creates the functions and tasks of the inside agent classes using the agents architecture diagram in this step. Functions and defined usages are result of several different architectures for the agents. They consist of reactiveness, designing, based on the user identification architecture and knowledge. In chain store system, the knowledge architecture which refers to mental state and the agents social Behavior is very suitable, because each of buyers and sellers interact with each other on the base of their goals and also the buyer and seller agents' reactiveness related to events occurred in system environment. They are response through the message. Reactiveness architecture is known suitable. Fig. 9 shows the functions and duties diagram of agent class of product price display.

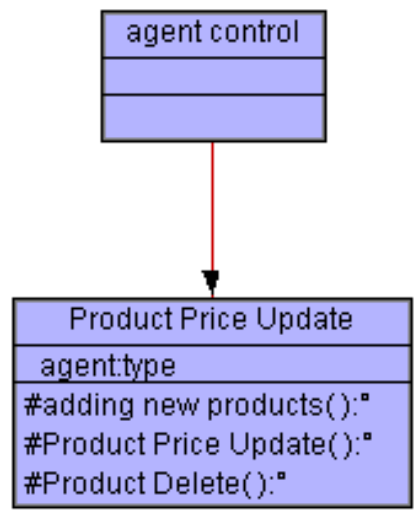

Fig9. Assembling Agent Classes Diagram in chain store system

\subsubsection{System Design}

The last step is the system design. It specifies the whole the structure of the agents in system. The outcome of this section is diagrams which determine the numbers, types and the locations of agents in system. The final result in a diagram is shown as deployment diagram. Fig. 10 shows the Deployment Diagram.

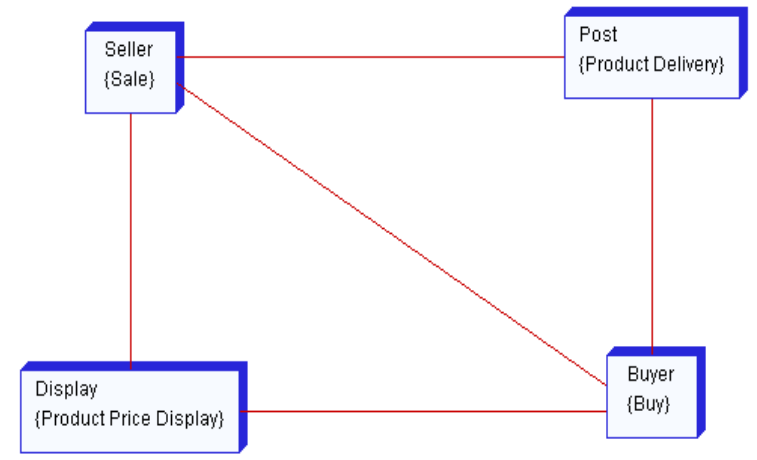

Fig10. Deployment Diagram in chain store system

\section{CONCLUSION AND FUTURE WORKS}

Because of the urgent need to the systems which have autonomous and independent ability of decision making. The importance of agent-oriented software engineering 
methodologies in this field shows that MaSE known as a multi agent methodology plays an important role in combining local behaviors with distributed decision making and autonomy. In this paper, we cover MaSE methodology in a practical system and show the sample system by MaSE graphic tools which area gent Tool. It can be said that this method acts well from the aspect of concept covering like : autonomy, mental aspects, concurrency and act-orientation and also from the aspect of covering the system life cycle such as: the requirement analysis, architectural design and implement and installation steps and pragmatic point of view like model accuracy, compatibility, the signs simplifications in system. Future works can be done are in field of Gaia methodology which is one of development methodologies of objectoriented software engineering. In this methodology, we can implement a system with the chain store system complexity to show the design and analysis steps of Gaia methodology in the frame of a system and have a more obvious understanding of this methodology.

\section{REFERENCES}

[1] Jennings N. R, 2000, On Agent-Based Software Engineering, Artificial Intelligence, Vol. 117, No. 2, pp. 277-296.

[2] B. Henderson, I. Gorton, 2002, Agent-based Software Development Methodologies, Summary of Workshop, ACM SIGPLAN International Conference on ObjectOriented Programming, Systems, Languages, and Applications (OOPSLA).

[3] N.R. Jennings, M. Wooldridge, Agent- Oriented Software Engineering, Queen Mary \& Westfield College University of London, London E1 4NS, United Kingdom. Available: http://icc.mpei.ru/documents/00000827.pdf. Last Available: 08,07,2012.

[4] C.A. Iglesias, M. Garijo, J.C. Gonzales, 1998, A survey of Agent-Oriented Methodologies, Proceedings of the 5th International Workshop on Intelligent Agents , Agent Theories, Architectures, and Languages (ATAL-98), pp. 317-330.

[5] S.A. DeLoach, 1999, Multiagent Systems Engineering: A Methodology and Language for Designing Agent Systems, Agent Oriented Information Systems (AOIS) '99, pp. 1-9.
[6] M.F. Wood, S.A. DeLoach, An Overview of the Multiagent Systems Engineering Methodology, In AgentOriented Software Engineering - Proceedings of the First International Workshop on Agent-Oriented Software Engineering, 10th June 2000, Limerick, Ireland. P. Ciancarini, M. Wooldridge, (Eds.) Lecture Notes in Computer Science. Vol. 1957, Springer Verlag, Berlin, January 2001.

[7] M. Wooldridge, N.R. Jennings, D. Kinny, The Gaia methodology for agent-oriented analysis and design, Kluwer Academic Publishers, Boston, Manufacttured in The Netherlands, pp. 1-27, Available: http://www.csc.liv.ac.uk/ mjw/pubs/jaamas2000b.pdf, Last Available: 02,06,2012.

[8] Wooldridge M. J., Jenning N. R. and Kinny D., 1999, A methodology for agent-oriented analysis and design , $3 r d$ international conference on Autonomous agents, pp. 6976.

[9] C. A. Iglesias, M. Garijo, J. Gonzalez and J. R. Velasco., 1998, Analysis and Design of multiagent systems using MAS-CommonKADS, Intelligent Agents IV: Agent Theories, Architectures and Languages, Singh, M. P., Rao, A. and Wooldridge, M.J., eds., Lecture Notes in Computer Science 1365.

[10] A.E. Arenas, G.B. Sanabria, 2002, Applying the MASCommon KADS Methodology to the Flights Reservation Problem: Integrating Coordination and Expertise, 5th Joint Conference on Knowledge-Based Software Engineering (JCKBSE 2002). No 39, Vol. 234, pp. 1-10.

[11] A. Tveit, 2001, A survey of Agent-Oriented Software Engineering, Norwegian University of Science and Technology, May 8.

[12] D.L. Scott, 2001, Analysis and Design using MASE and agent Tool, 12th Midwest Artificial Intelligence and Cognitive Science Conference (MAICS 2001) Miami University, Oxford, Ohio, pp. 1-7.

[13] S.DeLoach, M.Wood, C.Sparkman, 2001, Multi agent Systems Engineering, The International Journal of Software Engineering and Knowledge Engineering, Vol. 11 , No. 3, pp. 231-258.

[14] AgentTool, Online Available: http://macr.cis.ksu.edu/index.php?option=Com_content\& view $=$ article $\& i d=28$. Last Available: 30.03 .2012 . 